\title{
Acerca de las concepciones y prácticas de la educación en el sector solidario de Medellín, Colombia
}

Cristina Amariles-Mejía*

Magíster en Educación y Desarrollo Humano. Diseñadora de contenidos y docente virtual, Instituto Carlos Castillo (Odema), Buenos Aires, Argentina. Correo electrónico:

cristina.amariles@gmail.com

Recibido: 5 de febrero del 2015 Aprobado: 30 de abril del 2015

Cómo citar este artículo: Amariles-Mejía, C. (2015). Acerca de las concepciones y prácticas de la educación en el sector solidario de Medellín, Colombia. Cooperativismo \& Desarrollo, 23(106), 7-23. doi: http://dx.doi.org/10.16925/ co.v23i106.1125

\section{Resumen}

Introducción: la pregunta por las concepciones y prácticas de la educación en el sector solidario de Medellín es una apuesta por escuchar la voz de las personas y las organizaciones que han desarrollado este tipo de procesos. Metodología: esta investigación tiene un enfoque hermenéutico, con el fin de comprender los textos (entrevistas) de las personas clave en el sector de la economía social y solidaria en Medellín. Resultados: el artículo está dividido en cuatro partes: la primera, de contextualización del sector solidario en Medellín y de la investigación como tal; la segunda, centrada en las concepciones de la educación que resultan del análisis de las entrevistas; la tercera, está basada en las prácticas de la educación solidaria que presentan los entrevistados, y que permiten generar una tipología de prácticas según el enfoque y el lugar fundamental (o no) en el sector de la economía solidaria de Medellín y, finalmente, las conclusiones. Conclusiones: existen dos modos de ver la educación en el sector de la economía solidaria: uno, en relación con el fortalecimiento interno del sector y su apuesta educativa para su promoción y consolidación, y dos, la mirada articulada con los movimientos sociales y en una apuesta de transformación de la sociedad.

Palabras clave: cooperativismo, educación, sector solidario, solidaridad. 


\title{
Regarding Conceptions and Practices of Education in the Solidarity Sector of Medellín, Colombia
}

\begin{abstract}
Introduction: The question of educational conceptions and practices in the solidarity sector of Medellín is an initiative for listening to the voice of the people and organizations who have developed this type of processes. Methodology: This research has a hermeneutic approach aimed at understanding the text (interviews) of key individuals in the social and solidarity economy sector in Medellín. Results: The article is divided into four parts: the first provides context about the solidarity sector in Medellín and the research as such; the second focuses on conceptions of education that arise from analysis of the interviews; the third is based on the solidarity education practices demonstrated by those interviewed, generating a typology of practices, depending upon the approach and the fundamental place (or lack of one) in the solidarity economy sector of Medellín and, finally, the conclusions. Conclusions: There are two ways of seeing education in the solidarity economy sector: first of all, in relation to internal strengthening of the sector and its educational initiative for its promotion and consolidation; and secondly, in coordination with the social movements and initiatives for transformation of the society.
\end{abstract}

Keywords: cooperativism, education, solidarity sector, solidarity.

\section{Sobre as concepções e as práticas da educação no setor solidário de Medellín, Colômbia}

\section{Resumo}

Introdução: a pergunta pelas concepções e as práticas da educação no setor solidário de Medellín é feita a fim de que a voz das pessoas e das organizações que têm desenvolvido esse tipo de processos seja escutada. Metodologia: esta pesquisa tem um enfoque hermenêutico com o objetivo de compreender os textos (entrevistas) de pessoas-chave no setor da economia social e solidária em Medellín. Resultados: este artigo está dividido em quatro partes: primeira, de contextualização do setor solidário em Medellín e da pesquisa em si; segunda, centralizada nas concepções de educação que resultam da análise das entrevistas; terceira, baseada nas práticas de educação solidária que os entrevistados apresentam, o que gera uma tipologia de práticas que depende do enfoque e do lugar fundamental (ou não) no setor da economia solidária de Medellín e, finalmente, as conclusões. Conclusões: existem dois modos de ver a educação no setor da economia solidária: um, com relação ao fortalecimento interno do setor e sua aposta educativa para sua promoção e consolidação; e dois, o outro olhar em articulação com os movimentos sociais e numa aposta de transformação da sociedade.

Palavras-chave: cooperativismo, educação, setor solidário, solidariedade. 


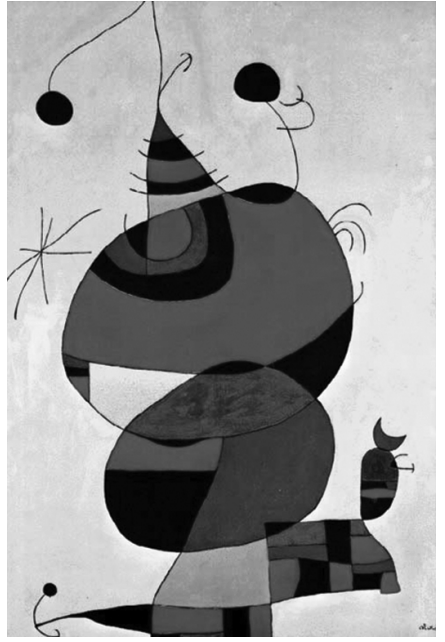

Desde la ventana más alta de mi casa, con un pañuelo blanco digo adiós a mis versos, que viajan hacia la humanidad.

Y no estoy alegre ni triste.

Ése es el destino de los versos.

Los escribí y debo enseñárselos a todos porque no puedo hacer lo contrario, como la flor no puede esconder el color,

ni el río ocultar que corre, ni el árbol ocultar que da frutos. He aquí que ya van lejos, como si fuesen en la diligencia,

y yo siento pena sin querer, igual que un dolor en el cuerpo.

¿Quién sabe quién los leerá? ¿Quién sabe a qué manos irán?

Flor, me cogió el destino para los ojos. Árbol, me arrancaron los frutos para las bocas. Río, el destino de mi agua era no quedarse en mí.

Me resigno y me siento casi alegre, casi tan alegre como quien se cansa de estar triste. ¡Idos, idos de mí!

Pasa el árbol y se queda disperso por la Naturaleza.

Se marchita la flor y su polvo dura siempre. Corre el río y entra en el mar y su agua es siempre la Mujer, pájaro y estrella, 1970. Joan Miró que fue suya.

Paso y me quedo, como el Universo.

El guardador de rebaños

Fernando Pessoa, publicado en su heterónimo Alberto Caeiro

\section{Contextualización del sector solidario en Medellín}

Este artículo forma parte la investigación: “Concepciones y prácticas de educación en el sector solidario de la ciudad de Medellín". Pretende dar cuenta de sus principales hallazgos y presentar los aciertos, retos y dificultades de una educación diferente con énfasis en la solidaridad; una educación que, al igual que el poema de Pessoa, pueda volar y generar posturas distintas y acciones diversas; una educación que contribuya a la formación de sujetos singulares y contextualizados, responsables de sí y de los otros. El sector solidario en Colombia cuenta con cerca de cuatro millones de asociados, lo que equivale aproximadamente al $10 \%$ de la población; genera trabajo a unas 600000 personas, lo que equivale al $4 \%$ de las personas ocupadas, con un 5,6 del PIB nacional y una irrigación del $10 \%$ del crédito total. Se estima que existen alrededor de 8800 organizaciones solidarias, ubicadas en 900 municipios del país (Valencia, 2012, p. 5).

En Antioquia, el sector de la economía solidaria se ha venido fortaleciendo en los últimos diez años, no solo con las organizaciones formales visibilizadas por la legislación (cooperativas, mutuales y fondos de empleados), sino también con otros procesos económicos populares que buscan ganar espacio y reconocimiento desde una apuesta solidaria. Esto hace que el sector de la economía solidaria sea complejo, por la diversidad de actividades económicas y el tamaño empresarial, y son los fundamentos y principios solidarios los que le dan identidad. Según las estadísticas de la Unidad Administrativa Especial de Organizaciones Solidarias (2012), Antioquia cuenta con 638 cooperativas que representan el $9 \%$ de estas entidades a nivel nacional, 94 mutuales que dan cuenta del $32 \%$ y 244 fondos de empleados que significan el $11 \%$ de los fondos de Colombia.

La economía solidaria está compuesta por dos pilares fundamentales: el económico y el solidario. Coraggio (2011) presenta el económico como un sistema de procesos de producción, distribución,

\footnotetext{
1 Requisito para optar al título de Magíster en Educación y Desarrollo Humano, de la Universidad de Manizales y el Cinde. Colombia, 2014. La investigación completa la pueden encontrar en este enlace: http://ridum.umanizales.edu.co:8080/jspui/bitstream/ 6789/1890/1/CONCEPCIONES\%20Y\%20PR\%C3\%81CTICAS\% 20DE\%20LA\%20EDUCACI\%C3\%93N\%20EN\%20EL\%20SECTOR\%20SOLIDARIO\%20DE\%20LA\%20CIUDAD\%20DE\%20 MEDELL\%C3\%8DN.pdf
} 
circulación y consumo que, por medio de principios, instituciones y prácticas, organiza en cada momento histórico a las comunidades y las sociedades para obtener las bases materiales de resolución de las necesidades y los deseos legítimos de todos sus miembros, actuales y futuros, de modo tal que permita la reproducción y el desarrollo de la vida, sosteniendo los equilibrios psíquicos e interpersonales entre las comunidades y la naturaleza.

El pilar solidario no es entendido como la acción que viene a resarcir carencias, penurias o escasez, sino como la que potencia el vínculo social, enriquece la calidad de vida y puede ser ofrecida y recibida no solo en momentos de pobreza, sino también de bienestar, para fortalecer a las comunidades y a los colectivos. En este sentido, la economía solidaria es una apuesta económica colectiva para el beneficio de una comunidad, cuyo centro son las personas y no la acumulación del capital. La solidaridad no pretende compensar los efectos de un negocio y entregar beneficios al final de la actividad económica, sino incidir en todo el circuito económico; que "la solidaridad se introduzca en la economía misma, y que actúe y opere en las diversas fases del ciclo económico, o sea, en la producción, circulación, consumo y acumulación" (Razeto, 1993, p. 15).

La economía solidaria es una propuesta de propiedad colectiva en la cual los excedentes que genera la actividad económica se revierten en beneficios para el grupo de asociados y los territorios en los cuales se encuentra, con una apuesta clara por el desarrollo local. Es una propuesta democrática en la que las decisiones se toman colectivamente y se participa de manera activa en los procesos sociales. También tiene una práctica ecológica que parte del respeto por el medio ambiente en toda la cadena productiva, incluyendo el manejo de los residuos. Hay, además, una ética de respeto por el productor y el consumidor, por lo tanto, priman el comercio justo y las acciones en redes, circuitos y alianzas.

Esta otra economía se gesta en el contexto de la hegemonía capitalista: la primacía del mercado y la idea de que este puede proveer los mecanismos que le otorguen al individuo la libertad para maximizar sus utilidades, pero no garantiza que todos obtengan los mismos beneficios, por lo que genera exclusión, pobreza y concentración de riqueza. Se da un predominio de lo económico sobre lo político, lo social y lo ecológico para estar al servicio del mercado, en el que las grandes multinacionales y los monopolios, al concentrar el poder económico, inciden directamente en el control de los Estados.
Así, en este contexto adverso la economía solidaria le apuesta a visibilizar otros modos de relación con lo económico y a promoverlos desde la educación, como parte de sus principios. En todas las organizaciones de economía solidaria se realizan acciones de educación, y a partir del Decreto 2880 de 2004 las cooperativas colombianas están obligadas a invertir el 20\% de sus excedentes en programas de educación formal aprobados por el Ministerio de Educación. Por ello es importante investigar y analizar las concepciones y prácticas, con el fin de aportar a la construcción de una lectura crítica de lo que se ha adelantado hasta el momento, de las cuales puedan surgir alternativas que contribuyan a su mejoramiento y cualificación.

Así, la pregunta de investigación se plantea en los siguientes términos: ¿Cuáles son las concepciones y prácticas de la educación en el sector solidario de Medellín? A partir de esta pregunta se pretenden valorar las relaciones entre las concepciones y prácticas que existen en el sector alrededor de la educación, con la intención de aportar a la comprensión de la solidaridad y su relación con la educación, para la creación de programas o el mejoramiento de los ya existentes, de forma que se evidencie la importancia de la educación en el sector solidario, es decir que de dichos programas se espera mayor rigurosidad académica, voluntad política y estructura para generar mejores procesos, más acordes con la concepción de solidaridad que se promulga.

Como objetivo general se propone aproximarse a la comprensión de las concepciones y las prácticas de la educación en el sector solidario en Medellín, y como objetivos específicos: 1) analizar las concepciones de educación en el sector solidario de Medellín, 2) analizar las prácticas de la educación en el sector solidario de tal ciudad y 3) establecer relaciones entre las concepciones y prácticas de la educación en el sector de la economía solidaria.

En este camino se encuentran las voces de la experiencia y la práctica en la conversación con personas de la Cooperativa Financiera Confiar, quienes han tenido programas educativos sostenidos durante varios años; el Instituto Saberes Solidarios, que es una experiencia de formación en economía social y solidaria, y de la Confederación Confecoop Antioquia, que agremia a las cooperativas del departamento y ha planteado un modelo educativo para el sector; además, se genera un espacio de diálogo con expertos en procesos educativos cercanos al sector de la economía solidaria. Las personas entrevistadas se eligieron por su experticia en el tema y sus acciones para darle un 
lugar importante a la educación en el sector de la economía solidaria en Medellín.

Esta investigación tiene un enfoque hermenéutico, por lo tanto, más allá de la pregunta por la representatividad de una "muestra", la intención es analizar los textos (en este caso, entrevistas) para comprender las concepciones y las prácticas de la educación desde su mismo discurso. El ejercicio de investigación hermenéutica implica desalojarse (o al menos intentarlo), darle juego a la pregunta, al laberinto, a lo inconcluso:

Su superioridad consiste en convertir lo extraño en propio al no disolverlo críticamente ni reproducirlo acríticamente, al revalidarlo interpretándolo con sus propios conceptos en su propio horizonte. La traducción puede hacer confluir lo ajeno y lo propio en una nueva figura, estableciendo el punto de verdad del otro frente a uno mismo (Gadamer, 2002, p. 179).

Es por eso que, en este artículo, no se encontrará la referencia directa de las organizaciones, los impactos y las personas atendidas, las especificidades de cada práctica nombrada, el porcentaje de representividad de las experiencias, en fin, preguntas que no están enmarcadas en este ejercicio hermenéutico basado en la comprensión y en el análisis de los textos, que plantean preguntas y reflexiones a partir de los discursos de los entrevistas, que permiten comprender una realidad desde la palabra y en contraste con la teoría, a lo que se le da la importancia y rigurosidad en la medida en que son estas prácticas cotidianas y las concepciones las que finalmente enmarcan las cotidianidades de las organizaciones, las que realmente se viven en el día a día y dan cuenta de las apuestas y los sentidos de lo que se hace.

Construir un relato trae consigo la exigencia de respetar al entrevistado, mediando entre su postura y la del investigador, y realizando un análisis crítico a partir de los elementos propios de las entrevistas, la literatura revisada y el objetivo planteado. El texto escrito es un modo de conjugar las subjetividades y apuestas del ejercicio de investigación, además de dar cuenta del marco histórico, social y cultural en el cual el texto social se torna en objetivación; al respecto, Alvarado (1993) comenta: "Sólo desde allí podrá desentrañar y reconstruir el sentido oculto del texto" (p. 272).

En este camino se encuentran las voces de la experiencia y la práctica en la conversación con personas de la Cooperativa Financiera Confiar, quienes han tenido una preocupación por los programas educati- vos, y de la Confederación Confecoop Antioquia, que agremia a las cooperativas del departamento y ha planteado un modelo educativo para el sector; además, se genera un espacio de diálogo con expertos en procesos educativos cercanos al sector de la economía solidaria.

Las personas entrevistadas se eligieron por su experiencia en el tema y sus acciones para darle un lugar importante a la educación en el sector de la economía solidaria en Medellín.

Aunque es claro quiénes son los entrevistados, específicamente en los testimonios se mantiene la confidencialidad de las personas que hablan en cada uno, eliminando las referencias concretas; por ello se utiliza solo un código para enumerar las entrevistas (E1, E2, entre otros), ya que, como se mencionó antes, se le da prioridad al relato y a las concepciones allí incorporadas, más allá de la revisión directa de una experiencia.

\section{Acerca de las concepciones de la educación}

Las concepciones de la educación en el sector de la economía solidaria tienen diferentes matices y apuestas, y no hay un consenso entre los entrevistados; por consiguiente, las descripciones de este apartado dan cuenta de una reflexión que articula retos para un enfoque educativo, que no se podría decir que es la guía actual de las prácticas educativas del sector.

\section{Una educación crítica/popular}

Una mirada de la educación que aparece en las entrevistas, es más un horizonte de sentido y una crítica a la educación instrumental, lo que genera pistas frente a un enfoque propio de la educación en el sector de la economía solidaria, o una educación solidaria como se le suele llamar a estos procesos. Así, hay una importante distancia con una concepción instrumental de la educación, que no genera crítica y reproduce la hegemonía capitalista:

Yo creo que la transformación de una sociedad tiene que pasar por una lucha ideológica que transforme conciencias y apuntale gente para una acción democrática y sensata. Educar se volvió instruir, capacitar, y la cultura es una cosa cada vez más cercana al entretenimiento, entonces ya no hay criterio (E6) ${ }^{2}$.

2 Esta numeración da cuenta del registro de las entrevistas de la investigación, como una decisión de generar un texto con la fluidez de los discursos, sin la referencia directa de la persona que lo dice. 
Pensar en una sociedad y en una economía distinta implica, a su vez, pensar en una educación distin$t a$, pues esta no es lejana a la promoción de modelos alternativos de desarrollo; por el contrario, es un actor principal para la construcción, mediante la articulación de varios sectores:

Otra sociedad es posible porque otra economía es posible, porque otro pensamiento es posible, porque otra cultura es posible, porque otra educación es posible. Lo que prende el suiche de todo es la educación, para poder llegar a un mundo diferente, a otro mundo posible. ¿Por qué es posible? Porque hay una educación diferente, que marca un contexto y una epistemología distintas orientadas a lo colectivo y al bien común, y no al bien particular; eso es posible y se puede hacer desde el trabajo de lo humano (E1).

Esta concepción de la educación habla de un proceso continuo que permita al educando apropiarse críticamente de los saberes, actitudes y destrezas necesarias para comprender la realidad, penetrarla, transformarla, valorar su universo simbólico y darle sentido a los eventos y las circunstancias de su cotidianidad, mediante la promoción de un pensamiento propio que se produzca desde la existencia y no rehúya la contingencia y la relación directa con los asuntos propiamente humanos, sino que los afronte y los asuma:

Asunción o asumirse tiene otro sentido más radical cuando digo: una de las tareas más importantes de la práctica educativo-crítica es propiciar las condiciones para que los educandos en sus relaciones entre sí y de todos con profesor o profesora puedan ensayar la experiencia profunda de asumirse. Asumirse como ser social e histórico, como ser pensante, comunicante, transformador, creador, realizador de sueños, capaz de sentir rabia porque es capaz de amar. Asumirse como sujeto porque es capaz de reconocerse como objeto. La asunción de nosotros mismos no significa la exclusión de los otros. En la "otredad" del "no yo" o del tú, la que me hace asumir el radicalismo de mi yo (Freire, 2008a, p. 42).

Por ello no se puede hablar de una educación neutral (Freire, 2008a): la educación tiene una intención, una búsqueda, una apuesta clara en las metodologías, en los contenidos y en su sentido mismo:

Si yo avalo la sociedad capitalista, su funcionamiento, su maquinaria, la manera en que opera, si me parece que eso está bien y que concierne a la mejor expresión de la humanidad, obviamente todas las prácticas educativas tratarán de afinar con ese modelo. Ahora, si yo tengo una distancia crítica respecto al sector capitalista, y pienso que el levantamiento de la sociedad sobre el trabajo asalariado/capital, y por ende que la explotación, no solamente no debe ser, sino que es posible que la humanidad se organice de otra manera, entonces mi práctica educativa debe apuntar a una visión y una concepción con fundamentos y razonamientos que mantengan un perfil crítico ante este modelo social, y educar para ir más allá y no solamente para la inmediatez de la práctica o de la eficacia (E6).

Educar en solidaridad es algo que trasciende la eficacia: implica vivir la democracia, concebir un mundo distinto, imaginar otras posibilidades en las que el otro realmente esté incluido; esto exige preguntarse por los sujetos y por el contexto en el cual están inmersos, lo que remite al enfoque sociocrítico que busca no solo la formación, sino también la transformación de realidades. Se lee la realidad para transformarla, con el objetivo central de construir un proyecto de sociedad justa y solidaria:

El humanismo es una concepción diferente a la concepción educativa de un modelo dominante que se convierte en aparato ideológico, es decir, el aparato dominante necesita una forma de educación; por eso creo que ninguna educación ni ningún modelo es inocente, tiene su intencionalidad (E1).

Aquí se establece la relación entre economía solidaria y educación, ya que los entrevistados, por formar parte del sector, constantemente hacen referencia a la educación como una posibilidad de pensar y generar acciones para que otra economía sea posible:

Pensar una educación distinta implica construir un modelo educativo igualmente distinto, debido a que en el discurso se escucha la percepción de una ideología amplia, de búsqueda de transformación desde la mirada estratégica; sin embargo al momento de las prácticas es necesario repensar cómo se traduce el propósito en una acción determinada (E3).

Debe ser una educación que converse, que forme parte del sector social y de sus búsquedas: "yo montaría, por ejemplo, una propuesta educativa que contara exclusivamente con la gente del sector social, gente 
comprometida y que tiene acumulada mucha experiencia" (E6). No es posible pensar la educación en el sector de la economía solidaria sin reconocer el camino que han recorrido las organizaciones sociales y de economía solidaria, y los espacios informales que han generado procesos educativos y que tienen mucho que aportar.

\section{Un diálogo con la academia y el mundo intelectual}

Las investigaciones, el mundo intelectual y el de la rigurosidad teórica deben dialogar con estas prácticas educativas, y aportar a la claridad del sentido, del cómo, de la apuesta misma de una educación diferente:

Cómo conectar el mundo de la realidad: quienes buscan la subsistencia o del desarrollo a través de todos los proyectos cooperativos y solidarios, y quienes se dedican a estudiar académicamente el mundo también solidario; yo pienso que estamos en esa búsqueda (E3).

En este sentido, Freire se suma a esta reflexión mediante el llamado a una educación que contribuya a las realidades y empodere a los sujetos frente a la construcción del conocimiento:

La metodología que defendemos exige, por esto mismo, que en el flujo de la investigación se hagan ambos sujetos de la misma, tanto los investigadores como los hombres del pueblo que, aparentemente, serían su objeto [...] Cuanto más asuman los hombres una postura activa en la investigación temática, tanto más profundizan su toma de conciencia en torno de la realidad y, explicitada su temática significativa, se la apropian (Freire, 2008b, p. 90).

La educación cuenta con varios campos y objetivos, y es necesario tenerlos claros para saber a qué se le está apuntando con los procesos, si a la formación o a la capacitación y al adiestramiento:

Los valores son la esencia originaria de la educación, y la educación se nutre de la formación en valores. El concepto de formación es un elemento central de la educación, porque tiene otros elementos como la capacitación, por ejemplo, y también está el adiestramiento, etc. Pero el centro de la educación es la formación de la persona, y ese centro se establece sobre la base de valores. Todo el proceso de educación que uno tiene desde el punto de vista formativo es formación en valores, sea desde que empieza a tener relacionamiento con el mundo externo, con la madre, con los padres, con los entornos familiares, y aún viejos, y siempre que se hable de formación en valores un valor central es la solidaridad. El ejercicio de la solidaridad y el entendimiento de la solidaridad por parte de los jóvenes es esencial para entender el relacionamiento en la sociedad, el compromiso con el otro, la ayuda al otro y el actuar conjunto; como valor, la gente debe entender ese concepto (E7).

En este sentido, Zuleta (2010) señala que:

La educación se ha convertido en la promoción de sujetos técnicos, en personas para el aparato productivo, pues solo reproduce la visión de un sistema cuyo principio es la acumulación de mayor capital, sin que interese el pensamiento y la creación real de las personas: La educación se ocupa de preparar a los estudiantes para intervenir en las distintas formas de trabajo productivo en los diversos sectores de la economía. Así, la eficacia de la educación para preparar los futuros obreros, contabilistas, ingenieros, médicos o administradores, se mide por las habilidades que el individuo adquiera para realizar tareas, funciones $u$ oficios dentro de un aparato productivo o burocrático (p. 74).

Este enfoque educativo debe apartarse de una academia que se olvida de lo político, de lo cultural, de las preguntas de los movimientos sociales y de las experiencias mismas de las organizaciones de economía solidaria, para hacer de la educación un relato riguroso que realmente contribuya a una sociedad más incluyente y, en esa vía, no sea posible replegarse de las tragedias del mundo global y de los abusos del sistema capitalista. Debe distanciarse de la formación centrada en las competencias y en el mejoramiento del hacer que olvida la comprensión del sentido, la sensibilidad y las artes: "el camino no puede ser la educación por competencias que viene promoviendo hoy el sistema tradicional; tiene que ser otra cosa y tiene que cruzarse con el escenario de la cultura, la literatura, las artes" (E4). Como afirma el entrevistado, otro apunte del enfoque tiene que ver con cómo articularlo con el arte, la estética y la cultura en general, no necesariamente para ser artista, sino para mirar el mundo con unos ojos distintos a los de la programación academicista de los resultados y la producción. 


\section{La potenciación de nuevas subjetividades}

Es importante mencionar que una educación en el sector de la economía solidaria debe fomentar la reflexión y el pensamiento crítico, que van más allá de seguir el camino de una sociedad dirigida por el capital, y pensar en la democracia, la inclusión y la dignidad:

A mi juicio, cultivar la capacidad de reflexión y pensamiento crítico es fundamental para mantener a la democracia con vida y en estado de alerta. La facultad de pensar idóneamente sobre una gran variedad de culturas, grupos y naciones en el contexto de la economía global y de las numerosas interacciones entre grupos y países resulta esencial para que la democracia pueda afrontar de manera responsable los problemas que sufrimos hoy como integrantes de un mundo caracterizado por la interdependencia. Y la facultad de imaginar la experiencia del otro (capacidad que casi todos los seres humanos poseemos de alguna manera) debe enriquecerse y pulirse si queremos guardar alguna esperanza de sostener la dignidad de ciertas instituciones a pesar de las abundantes divisiones que contienen todas las sociedades modernas (Nussbaum, 2011, p. 30).

Por lo tanto, debe promover sujetos que participan, preguntan y se proyectan. Así, los procesos educativos no solo ponen su centro en los contenidos y en lo que se debe saber, sino también en las personas y en cómo se construye conocimiento, por lo que sus voces y sus inquietudes son fundamentales para este propósito:

En una lógica solidaria la educación tiene que incorporar esa ideología y ese sistema de valores que promueve, y aterrizarlo en prácticas concretas. ¿De qué se ha nutrido esa lógica de la educación en el sector de la economía solidaria? Del diálogo de saberes, de los principios de la educación popular, del interaccionismo simbólico, del constructivismo y de las teorías críticas en general, que se preguntan también por el sujeto; no es simplemente que sea muy solidario, es más complejo que eso: ubicamos la posibilidad de que ese sujeto sea autónomo, emancipado, libre, con derechos (E2).

Lo anterior implica darle al sujeto un lugar como actor social, es decir, como un interlocutor válido con quien se construye el saber. Esto requiere de educadores abiertos a los saberes previos de las personas, a la crítica, a la diferencia, con el fin de evidenciar en la práctica educativa misma el significado de reconocer al otro y promover su emancipación. En este sentido, Nussbaum (2011) habla de la importancia de potenciar desde los escenarios educativos una mirada del otro con iguales derechos y obligaciones.

\section{Centralidad de las artes, la cotidianidad y los sueños}

La educación para humanizar la humanidad está atravesada por el encuentro con las artes:

A mí a veces me da dificultad decir cuál es el modelo a seguir, pero pensaba, por ejemplo, que la sensibilidad frente a las formas más amplias de cultura y de arte, la apreciación artística, para saber que hay unos loquitos por allá intentando trascender la miseria humana a través del arte y que el arte muestra más esas miserias que todas las glorias, pero que en esa filosofía uno se va encontrando porque nos sensibiliza distinto con la naturaleza (E3).

Respecto a los anteriores cuestionamientos, Nussbaum (2008) estima que la educación debe estar conectada con las humanidades y las artes, para que el sujeto pueda cultivar la compasión en tanto emoción dirigida al infortunio o al sufrimiento de otra persona y favorecer una relación más estrecha con el otro y consigo mismo:

Si el verdadero choque de las civilizaciones reside, como pienso, en el alma de cada individuo, donde la codicia y el narcisismo combaten contra el respeto y el amor, todas las sociedades modernas están perdiendo la batalla a ritmo acelerado, pues están alimentando las fuerzas que impulsan la violencia y la deshumanización, en lugar de alimentar las fuerzas que impulsan la cultura de la igualdad y el respeto. Si no insistimos en la importancia fundamental de las artes y las humanidades, estas desaparecerán, porque no sirven para ganar dinero. Sólo sirven para algo mucho más valioso: para formar un mundo en el que valga la pena vivir, con personas capaces de ver a los otros seres humanos como entidades en sí mismas, merecedoras de respeto y empatía, que tienen sus propios pensamientos y sentimientos, y también con naciones capaces de superar el miedo y la desconfianza en pro de un debate signado por la razón y la compasión (Nussbaum, 2011, p. 189). 
Otro elemento es la reflexión constante, el pensar que trasciende el hacer, el preguntarse, el cuestionar. Educar para soñar, pensar, buscar, crear y divertirse:

El enfoque de desarrollo, más que asistencialista, es un enfoque de pensamiento que pretende impulsar personas que reflexionen y piensen, más allá de la capacitación para el trabajo y las manualidades, con el fin de que la gente reflexione sobre sí misma, se busque... Es ese tema de ir buscando asuntos de ética en la vida cotidiana (E3).

Es la posibilidad de reflexionar en la cotidianidad misma, en los escenarios informales, en el encuentro con el otro, y no necesariamente en un espacio educativo formal, pues la educación no solo transcurre en las aulas de clase, sino que también tiene lugar en los entornos comunitarios, barriales y familiares. Ello implica hacer de la vida misma un escenario educativo para la complementación constante entre la teoría y la práctica, y para generar conocimiento desde las experiencias, validar el saber popular, aprender de los errores y construir nuevas realidades:

Lo educativo siempre está inmerso en la cotidianidad de la construcción misma, en los espacios naturales de reflexión que va teniendo la gente, en el solo hecho de que haya un acto de reflexión para repensar lo que se está haciendo y sus impactos. Cuando hay un proyecto y la gente lo conversa, lo reflexiona, de por sí ya hay un espacio educativo (E3).

El ocio creativo también es importante para este enfoque, pues abre las posibilidades a nuevos encuentros y formas de pensar el tiempo:

Esta sociedad del capital nos puso a pensar en el sentido del trabajo, y el sentido del trabajo es estar todo el tiempo ocupado, produciendo. Pero enseñar a vivir también implica preguntarse: ¿Cuál es tu tiempo de ocio? ¿Cuál es tu tiempo para el arte? (E3).

Como un modo de cerrar este apartado de las concepciones de la educación, los entrevistados brindan varios elementos para la reflexión y la construcción de una propuesta educativa propia, más cercana a la apuesta misma de una sociedad solidaria. En ese sentido, el enfoque del que hablan es el sociocrítico, caracterizado por ser una educación para la transformación y el cambio de la sociedad, que le da un lugar fundamental a los educandos y promueve sujetos emancipados, críticos y autónomos. Es una educación que saca la reflexión de las aulas de clases y la pone en la experiencia misma, en la cotidianidad, se aleja de la educación enciclopédica y la recrea en la vida, en los escenarios comunes. Este tipo de educación va más allá de las competencias, recupera el lugar de la palabra del otro, su saber y experiencia, y busca estar al servicio de los sectores sociales. Es un enfoque educativo con una postura que dista del modelo de sociedad promovido por el capitalismo, y en su lugar promueve una sociedad más solidaria, incluyente y digna para todos. Parte de una educación humanista que permite el diálogo escuela-comunidad, y que se piensa y se proyecta desde propuestas colectivas y de beneficio común, en las que las artes, la historia y la filosofía son invitadas fundamentales para reconocer la sensibilidad, la profundidad y la rigurosidad; a su vez, recoge, conversa y construye con las propuestas educativas de los movimientos sociales, sus luchas y avances.

\section{Prácticas de la educación solidaria}

Después de esta mirada amplia de la educación y de las apuestas por un enfoque crítico, transformador y emancipador, es necesario ver la realidad, cómo se están dando estos procesos educativos en las prácticas cotidianas y cuáles son los énfasis de estas. En las entrevistas aparecen prácticas concretas, acciones que dan cuenta de la cercanía o lejanía con lo planteado alrededor del sentido de la educación, y un posible enfoque pedagógico para este tipo de procesos. Son apuestas por la solidaridad como principio, valor y acción deseada en el mundo contemporáneo. Es de anotar que la construcción teórica alrededor de las prácticas tiene propósitos relacionados con una enseñanza sistematizada en la que interviene un nivel de reflexión o de análisis; la práctica, en suma, va más allá de la acción, y considera la rigurosidad desde la planeación, el seguimiento y la evaluación de los procesos. En este sentido, es preciso aclarar que en el presente texto las prácticas educativas se asumen como las acciones que adelantan las organizaciones de la economía solidaria en torno a la educación.

A partir de las entrevistas es posible agrupar las prácticas educativas del sector de la economía solidaria en nueve categorías: 1) educación en economía solidaria, 2) educación desde la cotidianidad, 3) procesos educativos comunitarios apoyados por las organizaciones de economía solidaria, 4) prácticas educativas 
para la comunidad educativa desde las organizaciones de economía solidaria, 5) educación para la transformación, 6) modelo pedagógico solidario, 7) educación obligatoria, 8) educación por competencias, y 9) educación sin un lugar claro en las empresas de economía solidaria. A continuación se describirá cada una de ellas.

\section{Educación en economía solidaria}

Esta práctica educativa es un llamado del sector de la economía solidaria a la promoción de una apuesta distinta, para lo cual es necesario sensibilizar a más personas sobre las especificidades de esta apuesta y cómo se evidencia en las empresas solidarias.

En los testimonios se habla de la posibilidad de formalizar las propuestas educativas que se han adelantado en la formación de líderes para la organización de proyectos asociativos desde el énfasis de la economía solidaria, las cuales tienen muchas afinidades con las propuestas educativas del sector social. Este proceso ha sido aislado, por lo cual se hace necesario tener acciones de mayor confluencia que puedan aportar a procesos de largo plazo, lo que evidencia que esta práctica aún está en un estado inicial y requiere de mayor rigurosidad y seguimiento:

Lo que pasa es que no hemos formalizado la educación. Lo que estamos intentando con Cultura y con Escuela es hacer ciclos que nos permitan pensar en una formación más formalizada, por decirlo de alguna manera, sobre todo en Escuela, mediante cursos básicos en economía solidaria, por ejemplo. Todo ese aparato que hemos venido construyendo nace también de esa experiencia más bien informal y de la práctica educativa que hemos tenido en el movimiento social, entonces yo creo que en este proceso lo que estamos intentando es surgir, porque creo que todavía estamos en proceso de parto de estos proyectos, como proyecto educativo, y no hemos llegado a un nivel de madurez; estamos en la fase de ensayo y error, entonces estamos probando: qué es, qué no es, por dónde es (E2).

La educación en economía solidaria y la formación política de las organizaciones sociales tienen puntos en común, y por eso el llamado es a construir procesos conjuntos que se enriquezcan de la experiencia informal de la educación en cursos, diplomados y talleres, en los cuales existe una riqueza importante que puede dar mayores luces al momento de generar nuevas apuestas educativas.

\section{Educación desde la cotidianidad}

Este tipo de prácticas evidencian el sentido de la educación desde los espacios cercanos, la vida misma en las empresas de economía solidaria y, a su vez, el diario vivir de las personas; ello demuestra que la solidaridad no se da en un momento o espacio específico, sino que incorpora todas las acciones de los sujetos, su intimidad, su vida privada y la acción pública.

En esta práctica se le da valor a la cotidianidad y a las acciones diarias que propician la reflexión. En este orden de ideas, la educación debe salir del aula de clase y los espacios formales para incorporarse a la dinámica de las personas y que desde allí puedan hacerla más propia, por lo que se hace preciso darle cabida al diálogo y a la reflexión desde lo cotidiano, e insistir en que la educación y la solidaridad no son asuntos dados sino que requieren esfuerzos, equivocaciones y retos.

Eso es como pedagogía desde la cotidianidad, en la medida que se hacen reuniones de los equipos de empleados, de la gente, o por el mismo revoleo que uno a veces puede hacer con los delegados y otros asociados. Las microreuniones que se han hecho públicamente para rendir cuentas son otra manera de decir que la información no se esconde sino que se publicita y se conversa (E3).

Estas prácticas valoran las acciones que adelantan las organizaciones solidarias para promover otros valores y para generar ambientes de aprendizaje desde la música, la poesía, la escritura, lo cual se puede hacer en momentos informales, reuniones y actividades espontáneas de las mismas organizaciones, como un modo de generar preguntas y de fomentar la reflexión sobre la solidaridad en cualquier momento de la vida.

\section{Procesos educativos comunitarios apoyados por las organizaciones de economía solidaria}

A estas prácticas se les da un valor especial, pues son propuestas que crecen en el seno de la organización comunitaria y, en ese sentido, tienen mayores ventajas para su consolidación y sostenimiento en el tiempo. Lo que hacen las organizaciones de economía solidaria es aportar a dicho fortalecimiento desde la interlocución, la construcción conjunta y el apoyo económico y administrativo. 
Estas prácticas materializan una combinación entre las intenciones de las organizaciones y los deseos de las personas, y en este sentido las entrevistas revelan que las apuestas de estas y de las organizaciones pueden generar mayor impacto y tener sostenibilidad en el tiempo, debido a que hay quién las piense, se identifique con ellas y las recree constantemente:

El proyecto de ahorro escolar, que se ha sostenido durante veinticuatro años, ha sido el intento de conectar un proyecto cooperativo con un proyecto de educación formal, mediante conversaciones con maestros, padres y estudiantes. Puede haber tenido altibajos, pero lo cierto es que se ha sostenido en el tiempo como una propuesta pedagógica y se ha ido retroalimentando. Yo veo claridades, entre ellas la construcción colectiva, y me alegra tener un grupo de personas que estén pensando el tema de cultura y ahorro con los maestros, y que las instituciones nos sigan buscando; como quien dice, 'venga enséñenos esa metodología, y hagámoslo juntos (E3).

Estas prácticas reconocen y resaltan el pensar y construir con los otros como un asunto fundamental a la hora de realizar proyectos. Las organizaciones pueden apoyar proyectos, pero es necesario que el protagonismo sea de los actores que les dan vida.

Yo creo que los proyectos necesitan una mirada que se fije, que profundice como un telescopio y que finalmente dimensione. Por ejemplo, el proyecto de ahorro escolar a mí me parece bellísimo; nació porque alguien nos tocó la puerta, obviamente venía construyéndolo y lo que necesitaba era una entidad que lo acompañara para poder redimensionarlo. (E3)

\section{Prácticas educativas para la comunidad educativa desde las organizaciones de economía solidaria}

Estas prácticas son apuestas de las organizaciones encaminadas a promover la solidaridad entre las nuevas generaciones, mediante la sensibilización frente al trabajo asociativo desde la experiencia misma de la escuela, con la intención de que los niños y jóvenes puedan promover relaciones más solidarias, incluyentes y transformadoras.

Estas prácticas educativas tienen el reto de comprender las dinámicas propias de los territorios, en este caso de la configuración social de una ciudad como Medellín, con el fin de proponer procesos for- mativos acordes con las necesidades y que realmente le aporten a la transformación. Es necesario que las prácticas educativas contemplen esto, lo hagan parte del proceso, lean las realidades y a partir de allí se planteen proyectos educativos contextualizados que reconozcan a los sujetos y a su vez les brinden opciones.

Hemos desarrollado una metodología de educación enfocada al tema de la solidaridad, y por eso tenemos muy desarrollado el tema de metodologías experienciales que resaltan el trabajo en equipo y el de escuchar e identificar las afinidades de los estudiantes, y también de las comunidades donde ellos están ubicados, porque la mayoría son comunidades vulnerables de la ciudad que han aprendido a vivir bajo el lema "sobrevive el más fuerte y sálvese quien pueda", entonces desde estos proyectos hemos tratado de trabajar y salvar un poco el trabajo colectivo de los muchachos (E5).

A su vez, llegar a un contexto de educación formal como la escuela también requiere de una lectura de las dinámicas propias de las nuevas generaciones, que están invadidas por la promoción del éxito individual y el consumismo; esto, sumado a un modelo escolar que no fomenta la solidaridad desde el trabajo colectivo, genera dificultades a la hora de promover un proceso educativo distinto basado en la asociatividad y la solidaridad:

Hemos percibido que ha sido una educación muy enfocada en lo individual, donde los estudiantes trabajan solos o tienen iniciativas de empresa y emprendimiento solos; de alguna manera es como si el modelo tradicional de educación llevara a que efectivamente los individuos seamos eso, seres individuales, entonces desde la educación que promueve un enfoque de solidaridad estamos tratando de que los estudiantes socialicen esas necesidades que tienen, esas ideas, esos proyectos, y se den cuenta de que trabajando juntos pueden lograr sus objetivos individuales y los objetivos colectivos, pues no son solo ellos los que tienen esa idea o esa necesidad, sino que es un grupo y alrededor de ese grupo ellos crecen, construyen y cumplen metas y sueños (E5).

Una propuesta de educación en el sector de la economía solidaria en un contexto individualista requiere de un esfuerzo mayor para generar procesos asociativos, y que se piense la solidaridad como 
una opción; es una apuesta a contracorriente de lo que vende el mercado a cada instante, de un contexto agresivo que ha permeado todas las esferas, incluida la escuela.

\section{Modelo pedagógico solidario}

Estas prácticas están enmarcadas en un modelo construido de manera colectiva por varias organizaciones de economía solidaria, con la intención de fomentar mayor seguimiento y coherencia interna para pensar las empresas que siguen este tipo de economía y desde allí direccionar procesos de formación para distintos públicos, pensando, especialmente, en la promoción de su sentido entre los asociados y dirigentes de sus organizaciones.

Este modelo pedagógico cuenta con un seguimiento continuo de las prácticas educativas, a partir de la apuesta por un enfoque específico para la constitución de organizaciones de economía solidaria:

Es un modelo que ha sido intencionado para el sector ${ }^{3}$, buscando romper esos esquemas según los cuales la educación es solo un curso de cooperativismo, entonces se van para una hostería a capacitarse durante dos horas y el resto de tiempo se gasta en una piscina (E5).

Este modelo posibilita a las organizaciones de economía solidaria hacer un "menor esfuerzo"; sin embargo, este no debería ser el sentido de una práctica educativa, ya que es necesario trascender las acciones que giran alrededor de "fidelizar al cliente", e ir en la vía de asumir como propio un referente pedagógico más amplio que le dé línea a sus prácticas:

Cuando las entidades lo conocen, digamos que se libran del esfuerzo, pero a la hora de implementar el resultado del modelo como material pedagógico es cuando se ve un poco la dificultad, porque rompe con los paradigmas y con lo que ellos ya traen tradicionalmente, que es lo que hace un comité de educación en una cooperativa: pensar los cursos y el gancho para atraer el asociado, que es el paseo, y pare de contar. El modelo les está planteando una ruta un poco más compleja, con estrategias, con proceso, con articulación, y ya hay que pensar un poco más cómo llevarlo a la cooperativa, lo que implica también inversión de tiempo, de recurso humano y de dinero. Entonces por ese lado sí hay un poco más de resistencia desde esos

3 Modelo educativo construido por Confecoop Antioquia. comités de educación, que están acostumbrados a hacer cosas más distintas y más materiales en el tema de la educación (E5).

Estas prácticas educativas buscan promover entre sus asociados el sentido solidario como organización empresarial, para que comprendan qué significa pertenecer a una empresa de economía solidaria. Esta búsqueda de identidad con el proyecto solidario es un reto de los procesos educativos:

La educación en las cooperativas debe ser un eje transversal y principal, porque así tendríamos asociados conocedores y convencidos del modelo, que se vincularían a una cooperativa no por un producto sino porque hace parte de una filosofía y un proyecto de vida (E5).

\section{Educación obligatoria}

Estas prácticas se centran en las organizaciones de economía solidaria que solo realizan acciones educativas para el cumplimiento legal de las veinte horas en formación requeridas para la constitución de las empresas. La educación no tiene un lugar importante, y por lo tanto no se hacen esfuerzos económicos ni políticos para constituir procesos educativos serios.

Estas prácticas consisten en la oferta de cursos de economía solidaria sin pedagogía ni didáctica, únicamente como un modo de cumplir un requisito legal:

Se hace una pregunta al esquema de formación de las cooperativas de los cursos de 20 horas, porque los veíamos como unos cursos muy esquemáticos, ortodoxos y muy salidos también del mundo al no analizar los contextos, no analizar políticamente o no relacionarnos con otros movimientos sociales (E3).

Así, hay prácticas dentro del sector solidario en las cuales la educación no tiene un lugar importante que permita resaltarla como algo a promover, y en las que es un asunto marginal a su estructura y a la asignación presupuestal para sus acciones: "En esos casos, la educación en el sector de la economía solidaria está prácticamente proscrita, o sea alejada, marginal" (E1). Esto lleva a la pregunta obvia de por qué hay pocas prácticas a resaltar en el sector de la economía solidaria, cuya repuesta es que la educación no es fundamental sino que el centro está en lo empresarial, con una división tajante entre hacer empresa y ser solidarios. 


\section{Educación por competencias}

Aquí se manifiesta el interés por apostarle al desarrollo de competencias, las cuales se centran en la adquisición de habilidades y destrezas técnicas para la competitividad. A esta formación por competencias, que es un modelo educativo mundial impuesto desde el Banco Mundial y el Banco Interamericano de Desarrollo, no se le hacen preguntas, por lo que no se generan procesos alternativos de formación sino que, por el contrario, la educación se centra en la exigencia de que las personas cuenten con unas competencias específicas:

Frente a este concepto general, que está muy capturado por las concepciones de la educación moderna y se ha llevado incluso a las cooperativas, donde hoy es muy normal hablar también de escenarios de formación por competencias, nosotros pensamos que definitivamente hay que tener una visión muchísimo más amplia, más integral, que empiece por tener una visión muy clara de la vida y del desarrollo en función de los seres humanos y no de la acumulación económica, porque desafortunadamente todo se ha convertido en mercancía y los bienes de mérito hoy están en función del negocio, como es el agua, como son las semillas, como es la salud, como es la educación; desafortunadamente, la gente no ve que ese es el modelo (E4).

Esta mirada de las competencias no permite abrir el espectro de la reflexión y se queda en una mirada técnica de lo educativo, sin que posibilite otro tipo de análisis.

\section{Educación para la transformación}

La educación para la transformación responde a la intención de hacer de la educación en el sector de la economía solidaria un proceso de mayor alcance, que aporte de manera decidida a la transformación de una sociedad marcada por la violencia, el miedo y la desconfianza en el otro, asuntos que impiden el establecimiento de relaciones más solidarias y de construcción colectiva:

Yo creo que se puede trascender un poco más, porque nosotros no nos podemos quedar en la técnica ni en creer que la educación cooperativa es un método para hacer empresa y no para hacer el movimiento ni para tener incidencia (E3).
Aspirar a una sociedad más solidaria implica el desarrollo de acciones encaminadas a una transformación desde procesos de mayor equidad e inclusión, por lo cual es necesaria la consolidación de un modelo pedagógico del que hay pocos asuntos escritos, aunque la conversación evidencia puntos comunes relacionados con el modo de hacer las cosas desde posturas políticas para el cambio social.

En esta medida, las prácticas que buscan ser alternativas se preguntan de manera constante si efectivamente están generando procesos distintos o su accionar no se diferencia del que caracteriza el modelo tradicional. La educación en el sector de la economía solidaria implica la construcción de dispositivos pedagógicos que permitan consolidar procesos asociativos en los cuales el otro tenga un lugar fundamental, un cambio que no se da en poco tiempo sino que requiere el esfuerzo de pensarlo, generar investigación e incluir en la conversación la economía y la educación:

Yo pienso que por lo menos lo intentamos, pero eso hay que hacerlo mucho más evidente, mediante la firma de acuerdos de compromiso de los actores en los que quede claro que esa es la propuesta, porque en la cotidianidad de la cooperativa no dejan de aparecer expresiones de la cultura tradicional, de las competencias, de los modelos que establecen que la formación está en las especializaciones y no en una visión integral. Por más que lo reflexionamos y lo decimos, en la práctica terminamos haciendo lo mismo otra vez. Ahora, hay un elemento que también condiciona que se termine haciendo lo mismo de la empresa tradicional: el marco regulatorio, no solamente de las cooperativas financieras sino también de las que están en el escenario de la salud, las de trabajo asociado, las de educación, que están muy condicionadas por el cumplimiento de una serie de normas, y por más que se quiera tener una educación más liberadora, más integral, más humanista, terminan capturadas en el corto plazo por la cultura del requisito (E4).

Entre las dificultades de hacer educación en el sector de la economía solidaria se encuentran las regulaciones del Estado y la normatividad internacional, ya que se prohíbe realizar acciones que no sean acordes con la actividad empresarial (educativas, por ejemplo), se define en qué se deben invertir los excedentes de la organización, no se reconoce la propiedad colectiva como un modo de organización de economía solidaria y se establecen los mismos 
requisitos legales de la propiedad privada, entre otros. Muchas de estas condiciones en ocasiones son un obstáculo para lograr procesos educativos rigurosos, y todo se centra en responder a este tipo de exigencias. Por lo tanto, también es un reto mediar entre los requerimientos externos y la convicción de que se está aportando a una sociedad distinta. Ignorar las formalidades puede hacer que un proyecto desaparezca, porque se convierte solo en una estrategia económica o porque se centra en los procesos educativos y se queda sin piso financiero para su ejecución.

\section{La educación sin un lugar claro en las empresas de economía solidaria}

Esta es la postura de muchas organizaciones que no le dan un lugar importante a la educación, por lo que sus prácticas son débiles, nulas o se reducen a la compra de servicios específicos; aunque se hable de la importancia de la educación, se queda en el nivel discursivo.

Lo anterior da cuenta de las contradicciones internas del sector de la economía solidaria en lo referente a la educación, pues en las entrevistas se hace un llamado a la importancia de la educación como uno de sus principios, pero las prácticas educativas son poco rigurosas y de bajo alcance:

¿Cuál es la educación que tenemos hoy en el cooperativismo? Una educación atomizada, dispersa, desconectada de los principios y los valores cooperativos, infiel a esos ideales cooperativos, aunque cada uno hace su mayor esfuerzo. Por eso ya el lenguaje común es competitividad, eficiencia, productividad, negocios, entonces se distorsiona porque primero monto el negocio y después creo el cooperativismo, o sea, primero creo la cooperativa y después creo el cooperativista; no, es primero el cooperativista como militante, ni siquiera como simpatizante, porque hay muchos simpatizantes pero pocos militantes. ¿Por qué los cooperativistas nos creemos seres diferentes, si el cooperativismo se expresa como se expresa la cultura? A no ser que creemos una contracultura... O sea, ¿qué es lo que nos da esa especialidad? Nada, porque estamos haciendo lo mismo (E1).

El hecho de que el sector solidario esté captado por la economía tradicional, cuyo centro es la rentabilidad, no da cabida a búsquedas alternativas serias en las propuestas educativas.
En esa medida, en el sector de la economía solidaria no se reconocen acuerdos ni reflexiones sobre el sentido de la educación y de desarrollar procesos educativos, lo que reduce su campo de acción a actividades puntuales. Las empresas de economía solidaria están pensadas como un aporte alternativo de la economía para una sociedad más incluyente, lo que no es claro en el análisis que hacen los entrevistados al relatar la realidad vivida:

En el caso colombiano no existen acuerdos. Nosotros los colombianos tendemos a obedecer lo que dice la ley, y la ley simplemente dice que es obligación hacer educación cooperativa o educación mutualista, sin claridad de cuáles son los ámbitos y cuáles los instrumentos (E7).

\section{Conclusiones}

Esta reflexión final está enmarcada en la lectura sobre las entrevistas y su relación con las concepciones y prácticas de la educación. Como se dijo inicialmente, no pretende ser una generalización, sino una invitación al debate y a continuar analizando de modo más riguroso el lugar de la educación en el sector, y los aportes de las experiencias al mejoramiento de lo que se viene construyendo.

La educación en el sector de la economía solidaria se mueve en un campo muy complejo, por estar en el marco de una acción empresarial; por ello suelen ser endebles los límites entre ser eficiente económicamente y ser una estrategia social y política. Las organizaciones de economía solidaria no son un fin en sí mismas, sino una herramienta; tener esta claridad permite diferenciar cuales son las prácticas educativas acordes con la economía buscada.

En suma, a partir de las entrevistas, en las cuales se evidencian nueve categorías de prácticas educativas en el sector de la economía solidaria de Medellín, se pueden señalar dos posturas: la educación como medio para el fortalecimiento de las organizaciones en sí mismas, y la educación como proceso para el cambio social. En el caso de la primera, las organizaciones de economía solidaria se preguntan por estrategias educativas centradas en el mejoramiento y la consolidación del sector solidario; en el caso de la segunda, las organizaciones están interesadas en promover la asociatividad y los procesos de solidaridad como una relación política, social y cultural, en la cual la educación se asume como la columna vertebral de un proceso más complejo de cambios en la sociedad. 
De acuerdo con las dos nociones de la educación - el campo propio de la economía solidaria y el sentido más amplio que se refiere a la transformación social-, las prácticas pueden estar más cerca o más lejos de cada búsqueda. En relación con el fortalecimiento del sector, es posible señalar capacitaciones técnicas, acciones en instituciones educativas, grupos de formación internos y estrategias de articulación con otras organizaciones de economía solidaria, que permiten evidenciar el avance hacia un modelo pedagógico cuyo objetivo es fortalecer la identidad de las organizaciones. Sin embargo, estás prácticas aún son débiles en cuanto a su continuidad, al reconocimiento de la importancia por parte de las mismas organizaciones, y es importante avanzar en este sentido, en la vía de procesos de mayor rigurosidad e impacto social.

La otra tendencia muestra prácticas articuladas con los movimientos sociales mediante acciones de mayor complejidad, con el propósito de generar transformaciones que vayan más allá del sector de la economía solidaria, y la convicción de que la educación le puede aportar a esta articulación; para ello, hay que tener en cuenta que no es solo un asunto educativo, sino que requiere mayor compromiso político, económico y cultural, con el fin de sostener una apuesta educativa distinta desde el protagonismo de las personas, las redes, la incidencia pública, la promoción de ciudadanías, entre otros. Sin embargo, estas acciones son más dispersas y poco sistematizadas, lo que no permite reconocer sus avances y retos más concretos.

Esta investigación permitió visualizar cómo la educación no tiene un lugar claro en el sector de la economía solidaria de Medellín, no es una apuesta directa desde el enfoque y las acciones concretas para su fortalecimiento. En las siguientes premisas se evidencian los diferentes énfasis de las prácticas encontradas:

- Son frecuentes las prácticas con poca estructura organizativa y con centralización de las labores en equipos de trabajo pequeños, lo que no les permite a las organizaciones encarnar la apuesta de educación que se pretende.

- Son prácticas con muchas claridades pedagógicas pero con poca sistematicidad del cómo hacerlo en la cotidianidad y llevar un seguimiento riguroso.

- Predomina un modelo pedagógico basado en el ofrecimiento de servicios educativos a las organizaciones de economía solidaria.
- Son prácticas educativas centradas en la capacitación técnica y la formación por competencias, con poco sentido político y una visión estrecha de la educación.

- Es un sector solidario sin claridades ni acuerdos alrededor del sentido de la educación, lo que se refleja en prácticas educativas dispersas y con poca contundencia.

- Las prácticas educativas en la ciudad promueven el emprendimiento con ciertos elementos asociativos, pero no hay claridad sobre su sentido ni se ofrecen como una opción real de inclusión y equidad.

- La reflexión, el análisis y la investigación de los procesos educativos no tienen un lugar importante, $y$ se puede ver reflejado en los pocos procesos investigativos, las mínimas publicaciones y los pocos documentos institucionales al respecto.

- La legislación nacional e internacional obliga a las organizaciones a centrarse en asuntos técnicos y a responder por los requisitos, lo que no deja lugar para pensar el proyecto solidario.

- Se dan prácticas educativas con la intención de "fidelizar al cliente", que no generan reflexión y pierden de vista el sentido mismo de la educación.

En algunos momentos las prácticas se ven afectadas por las realidades presupuestales de las instituciones y la necesidad de una estructura para sostener una propuesta tan amplia como se desee. En ellas son muy puntuales las acciones que aspiran a transformar la sociedad; la realidad organizacional impide contar con un equipo de trabajo fortalecido, se tiene sobrecarga laboral y se carece de la estructura operativa necesaria: "nunca hemos podido tener un docente de planta, y estos procesos necesitan gente que esté de lleno. Las funciones operativas son tantas y tan grandes, que muchas veces se tiene que priorizar lo urgente en lugar de lo importante" (E2). En el contexto actual, las condiciones de supervivencia y la precariedad laboral se ven reflejadas en la dificultad de desarrollar claridades ideológicas y de sentido para llevar a la práctica y generar un proceso real.

Pensar la práctica implica darse cuenta de lo que no se está haciendo bien y se debe mejorar, además de reconocer los alcances, ya que los procesos educativos son inacabados y no es posible alcanzar todos los cambios esperados. También se deben tener en cuenta las subjetividades, los contextos y el modelo hegemónico, y por eso es fundamental para las organizaciones reconocerse, generar preguntas y volver a pensarse: 
Algo no hacemos bien y tenemos que descubrir qué es, porque también tenemos una dificultad para hacernos entender y ahí hay una cosa que todavía no está resuelta. ¿Cómo sabemos cuál es la intencionalidad de un proyecto para que la gente se identifique más con él? Ahí hay enredos, nosotros también caemos en la trampa y hemos hecho unos modelos educativos instrumentalistas... ¿Cómo no hacerlos? La única manera es ser más analíticos, más pensantes. También tenemos que llevar una educación que no le muestre las respuestas a la gente, para que la gente se pregunte, porque estamos siendo conductistas (E3).

En este panorama se mueven las prácticas educativas del sector de la economía solidaria, lo alienta la necesidad de propuestas educativas más claras, ejercicios de sistematización de lo que se ha realizado hasta el momento, articulación entre las organizaciones para promover propuestas con mayor impacto, pero especialmente claridad en el enfoque de la educación y en que las prácticas realizadas vayan en pro de un objetivo claro, con un seguimiento y una evaluación constantes, lo que implica en sí misma una apuesta presupuestal y administrativa para su desarrollo.

Sin embargo, en el sector de la economía solidaria en Medellín no es visible la configuración de una propuesta de articulación social, política y económica de las organizaciones sociales. La educación es un eje fundamental al cual se podría dar mayores niveles de importancia para la promoción de una propuesta como la solidaria, que tiene en el centro a las personas y el mejoramiento de su calidad de vida. Y en esa vía, es necesario admitir que las propuestas económicas solidarias requieren acciones políticas y que las organizaciones sociales requieren de una acción más contundente en cuanto a la organización de sus economías, el consumo y el manejo de los recursos. Asimismo, es fundamental la articulación con sectores que tienen búsquedas afines, para hacer camino a pesar de las dificultades que entraña construir con otros.

La educación no es un agregado para pensar un mundo más solidario: es un motor que permite conversar, reflexionar, analizar y construir con otros. Un proyecto educativo solidario no puede aspirar a educar sujetos de una determinada forma, pues no es posible negar las subjetividades; por lo tanto, no busca la alienación sino la libertad de acción a partir de reflexiones claras y argumentos sólidos. La educación abre puertas, posibilita mundos, construye sueños. Nece- sita tocar las subjetividades, las intimidades, el mundo privado, para lograr incidir efectivamente en lo público. Los discursos de cambio mediante la movilización que se dan en los escenarios públicos se quedan sin piso cuando en lo íntimo se es cruel, se hace daño, se genera dolor; es necesario evidenciar esta preocupación, conversarla, ponerla en discusión, ya que muchas veces se hace un llamado a la necesidad de desarrollar una sociedad distinta con palabras vacías. Las sociedades están compuestas por sujetos seguros de sus posturas, pero poco dejan entrever los silencios y las soledades que impiden que la solidaridad realmente transforme sus vidas.

Educar en solidaridad en un mundo insolidario, egoísta, individualista, autista, consumista..., nunca será fácil. Reconocer la incoherencia y la decepción que generan este tipo de apuestas, poner de presente que para generar acuerdos colectivos requiere de renuncias individuales, todo esto es necesario a la hora de pensar una propuesta solidaria, no para renunciar, sino para poner todo el empeño en ir tras la utopía, que para eso sirve, para caminar.

\section{Referencias}

Alvarado, S. V. (1993). Investigación cualitativa. Confrontación y prospectiva. Antioquia: Universidad de Antioquia.

Corraggio, J. L. (2011). La economía social y solidaria. El trabajo antes que el capital. Quito: Ediciones Abya-Yala. Recuperado de http://www.coraggioeconomia.org/jlc/ archivos\%20para\%20descargar/economiasocial.pdf

Freire, P. (2008a). Pedagogía de la autonomía. Argentina: Siglo XxI.

Freire, P. (2008b). Pedagogía del oprimido. Argentina: Siglo XXI.

Gadamer, H. G. (2002). Verdad y método II. Salamanca: Ediciones Sígueme.

Nussbaum, M. C. (2008). Paisajes del pensamiento: La inteligencia de las emociones. Barcelona: Paidós.

Nussbaum, M. C. (2011). Sin fines de lucro: Por qué la democracia necesita de las humanidades. Bogotá: Katz Editores.

Razeto, L. (1993). Los caminos de la economía solidaria. Recuperado de http://www.luisrazeto.net/content/loscaminos-de-la-economia-de-solidaridad

Unidad Administrativa Especial de Organizaciones Solidarias. (2012). Plan estratégico 2012-2014. Recuperado de http://www.organizacionessolidarias.gov.co 
Valencia, L. E. (2012, 20 de marzo). Real opción para el desarrollo social. Periódico Desde Abajo: Suplemento de economía cooperativa y solidaria, 177, 3-6.
Zuleta, E. (2010). Educación y democracia: Un campo de combate. (O. B. Virtual, Ed.). Recuperado de file://C:/ Users/Operador\%20Internet/Downloads/educaciony-democracia.pdf 